\title{
CORRECTION
}

\section{Correction to: An analysis of scientometric data and publication policies of rheumatology journals}

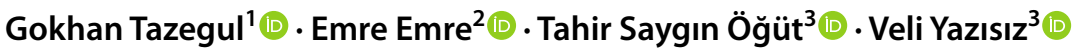

Published online: 22 July 2021

(c) International League of Associations for Rheumatology (ILAR) 2021

\section{Correction to: Clinical Rheumatology} https://doi.org/10.1007/s10067-021-05824-2

In the original published version of this article, the Tables were presented incorrectly. The corrected tables are shown as follows Tables 1, 2, 3 and 4:

The original article has been corrected.

The original article can be found online at https://doi.org/10.1007/ s10067-021-05824-2

Gokhan Tazegul

drgtazegul@gmail.com

1 Internal Medicine, Ankara Polatlı Duatepe State Hospital, Ankara, Turkey

2 Allergology and Immunology, Hatay Research and Training Hospital, Hatay, Turkey

3 Rheumatology, Akdeniz University School of Medicine, Antalya, Turkey 
Table 1 Bibliographic and scientometric data and publication policies of rheumatology journals

\begin{tabular}{|c|c|c|c|}
\hline \multirow[t]{3}{*}{ Types of published papers } & All types & & $14(63.6 \%)$ \\
\hline & All except case reports & & $7(31.8 \%)$ \\
\hline & Review only & & $1(4.6 \%)$ \\
\hline \multirow[t]{13}{*}{ Bibliometric data } & Citable articles & 2015 & $196(44-386)$ \\
\hline & & 2016 & $197(42-411)$ \\
\hline & & 2017 & $209(26-549)$ \\
\hline & & 2018 & $197(21-443)$ \\
\hline & & 2019 & $183(22-631)$ \\
\hline & Citation counts & 2015 & $4022(12-35393)$ \\
\hline & & 2016 & $5292(39-39185)$ \\
\hline & & 2017 & $5728(47-41994)$ \\
\hline & & 2018 & $6326(81-44754)$ \\
\hline & & 2019 & $6619(164-45886)$ \\
\hline & OA article publication rate & & $10.6(0-100)$ \\
\hline & OA article citation rate & & $12.6(0-100)$ \\
\hline & $\mathrm{C} / \mathrm{C}$ ratio & & $0.99(0.29-1.33)$ \\
\hline \multirow[t]{6}{*}{ Scientometric indexes } & JIF & & $3.17(0.73-16.1)$ \\
\hline & $\mathrm{CS}$ & & $4.4(0.7-25.9)$ \\
\hline & HI & & $78(10-305)$ \\
\hline & SNIP & & $1.05(0.325-3.89)$ \\
\hline & ES & & $0.0088(0.00046-0.073)$ \\
\hline & SJR & & $1.06(0.24-6.14)$ \\
\hline \multirow[t]{4}{*}{ Publishing policy } & Full OA & & $6(27.3 \%)$ \\
\hline & & With APC & $4(18.2 \%)$ \\
\hline & & Free of charge & $2(9.1 \%)$ \\
\hline & Hybrid OA & & $16(72.7 \%)$ \\
\hline \multirow[t]{2}{*}{ Publication fee } & Mandatory & & $9(40.9 \%)$ \\
\hline & Optional & & $11(50 \%)$ \\
\hline \multirow[t]{3}{*}{ Fee timing } & Before & & $0(0 \%)$ \\
\hline & After & & $19(86.3 \%)$ \\
\hline & Both & & $1(4.6 \%)$ \\
\hline OA APC $(n=20)$ & & (USD) & $3300(605-4700)$ \\
\hline
\end{tabular}

OA: Open access, $\mathrm{C} / \mathrm{C}$ ratio: ratio of citations to $\mathrm{OA}$ articles to the ratio of citable $\mathrm{OA}$ articles in journals with hybrid OA publication policy. JIF: journal impact factor, CS: CiteScore, HI: Hirsch index, SNIP: Source Normalized Impact per Paper, ES: Eigenfactor score, SJR: Scientific journal ranking. APC: Article Processing/Publishing Charge. USD: United States of America Dollars 
Table 2 Spearman's rho correlation coefficients of scientometric indexes of rheumatology journals

\begin{tabular}{llll}
\hline Scientometric indexes & Spearman's rs & $p$-value \\
\hline JIF & CS & 0.933 & $<0.0001$ \\
& HI & 0.656 & 0.001 \\
& SNIP & 0.867 & $<0.0001$ \\
& ES & 0.564 & 0.006 \\
& SJR & 0.917 & $<0.0001$ \\
CS & HI & 0.693 & $<0.0001$ \\
& SNIP & 0.939 & 0.0001 \\
& ES & 0.636 & 0.001 \\
& SJR & 0.974 & $<0.0001$ \\
HI & SNIP & 0.669 & 0.001 \\
& ES & 0.922 & $<0.0001$ \\
& SJR & 0.792 & $<0.0001$ \\
SNIP & ES & 0.669 & 0.001 \\
& SJR & 0.942 & $<0.0001$ \\
ES & SJR & 0.724 & $<0.0001$ \\
\hline
\end{tabular}

JIF: journal impact factor, CS: Citescore, HI: Hirsch index, SNIP: Source Normalized Impact per Paper, ES: Eigenfactor score, SJR:

Scientific journal ranking

Table 3 Distribution of rheumatology journals into quartiles according to six different scientometric indexes

\begin{tabular}{|c|c|c|c|c|c|}
\hline$\underline{\text { JIF }}$ & $\underline{\mathrm{CS}}$ & HI & $\underline{\text { SNIP }}$ & $\underline{E S}$ & $\underline{\text { SJR }}$ \\
\hline$\# 1$ & $\# 1$ & $\# 2$ & $\# 1$ & $\# 1$ & $\# 1$ \\
\hline$\# 2$ & $\# 2$ & $\# 1$ & $\# 2$ & $\# 2$ & \#2 \\
\hline$\# 3$ & $\# 4^{\mathrm{a}}$ & $\# 10$ & $\# 5$ & $\# 3$ & $\# 7$ \\
\hline$\# 4^{\mathrm{a}}$ & $\# 7$ & \#3 & $\# 7$ & $\# 6^{\mathrm{a}}$ & $\# 5$ \\
\hline$\# 5$ & $\# 3$ & $\# 7$ & $\# 3$ & $\# 7$ & $\# 3$ \\
\hline$\# 6^{\mathrm{a}}$ & $\# 5$ & $\# 6^{\mathrm{a}}$ & $\# 4^{\mathrm{a}}$ & $\# 20^{\mathrm{a}}$ & $\# 6^{\mathrm{a}}$ \\
\hline$\# 7$ & $\# 6^{\mathrm{a}}$ & $\# 5$ & $\# 13^{\mathrm{a}}$ & $\# 10$ & $\# 10$ \\
\hline$\# 8$ & $\# 8$ & \#16 & $\# 8$ & $\# 14$ & $\# 4^{\mathrm{a}}$ \\
\hline$\# 9$ & \#10 & \#11 & $\# 6^{\mathrm{a}}$ & $\# 11$ & $\# 8$ \\
\hline$\# 10$ & $\# 11$ & $\# 20^{a}$ & $\# 10$ & $\# 5$ & \#11 \\
\hline \#11 & $\# 13^{\mathrm{a}}$ & $\# 14$ & $\# 20^{\mathrm{a}}$ & $\# 16$ & $\# 13^{\mathrm{a}}$ \\
\hline \#12 & $\# 16$ & $\# 9$ & $\# 14$ & $\# 18$ & \#16 \\
\hline$\# 13^{\mathrm{a}}$ & $\# 12$ & $\# 12$ & $\# 11$ & \#19 & $\# 9$ \\
\hline$\# 14$ & $\# 14$ & $\# 18$ & $\# 12$ & \#8 & $\# 12$ \\
\hline$\# 15$ & $\# 9$ & $\# 8$ & $\# 16$ & $\# 9$ & $\# 20^{a}$ \\
\hline$\# 16$ & $\# 18$ & \#17 & $\# 9$ & $\# 17$ & \#14 \\
\hline \#17 & $\# 17$ & \#15 & $\# 17$ & $\# 13^{\mathrm{a}}$ & \#18 \\
\hline$\# 18$ & $\# 20^{\mathrm{a}}$ & $\# 19$ & $\# 19$ & $\# 12$ & $\# 17$ \\
\hline \#19 & \#19 & $\# 13^{\mathrm{a}}$ & \#18 & \#15 & \#19 \\
\hline$\# 20^{\mathrm{a}}$ & \#15 & $\# 4^{\mathrm{a}}$ & $\# 15$ & $\# 4^{\mathrm{a}}$ & \#15 \\
\hline$\# 21^{\mathrm{b}}$ & $\# 21^{\mathrm{b}}$ & $\# 21^{\mathrm{b}}$ & $\# 21^{\mathrm{b}}$ & $\# 21^{\mathrm{b}}$ & $\# 21^{\mathrm{b}}$ \\
\hline$\# 22^{\mathrm{b}}$ & $\# 22^{\mathrm{b}}$ & $\# 22^{b}$ & $\# 22^{\mathrm{b}}$ & $\# 22^{\mathrm{b}}$ & $\# 22^{b}$ \\
\hline
\end{tabular}

JIF: journal impact factor, CS: Citescore, HI: Hirsch index, SNIP: Source Normalized Impact per Paper, ES: Eigenfactor score, SJR: Scientific journal ranking. JIF was used as the reference scientometric index for the quartiles. The 1st quartile is presented in green, the 2nd quartile in yellow, the 3rd quartile in orange, and the 4th quartile in red. The superscript ${ }^{\text {a }}$ denotes OA journals with APC, and the superscript letter ${ }^{\mathrm{b}}$ refers to journals with a free OA publication policy 
Table 4 Comparison of bibliographic and scientometric data of rheumatology journals with all OA with APC and hybrid OA publishing policies

\begin{tabular}{|c|c|c|c|c|}
\hline & & & Hybrid OA $(n=16)$ & Full OA $(n=6)$ \\
\hline \multirow[t]{3}{*}{ Types of published papers } & All types & & $10(62.5 \%)$ & $4(66.7 \%)$ \\
\hline & All except case reports & & $5(31.3 \%)$ & $3(33.3 \%)$ \\
\hline & Review only & & $1(6.3 \%)$ & $0(0 \%)$ \\
\hline \multirow[t]{13}{*}{ Bibliometric data } & Citable articles & 2015 & $202(64-332)$ & $61(44-386)$ \\
\hline & & 2016 & $201(50-411)$ & $66(42-314)$ \\
\hline & & 2017 & $222(57-366)$ & $70(26-549)$ \\
\hline & & 2018 & $212(54-424)$ & $72(21-443)$ \\
\hline & & 2019 & $188(59-413)$ & $74(22-631)$ \\
\hline & Citation counts & 2015 & $4571(1002-35393)$ & $493(12-12302)$ \\
\hline & & 2016 & $5324(1416-39185)$ & $703(39-13730)$ \\
\hline & & 2017 & $6560(1661-41994)$ & $780(47-15259)$ \\
\hline & & 2018 & $7118(1674-44754)$ & $1053(81-16287)$ \\
\hline & & 2019 & $6818(1793-45865)$ & $1166(164-17130)$ \\
\hline & $\mathrm{OA}$ article publication rate & & $8.68 \%(0-32.2 \%)$ & 100 \\
\hline & $\mathrm{OA}$ article citation rate & & $7.63 \%(0-31.6 \%)$ & 100 \\
\hline & $\mathrm{C} / \mathrm{C}$ ratio & & $0.99(0.29-1.33)$ & 1 \\
\hline \multirow[t]{6}{*}{ Scientometric indexes } & JIF & & $3.33(1.98-16.1)$ & $2.23(0.73-5.04)$ \\
\hline & CS & & $4.4(2.1-25.9)$ & $3.85(0.7-8)$ \\
\hline & HI* & & $85(38-305)$ & $32(10-144)$ \\
\hline & SNIP & & $0.962(0.686-3.89)$ & $1.22(0.325-1.488)$ \\
\hline & ES & & $0.0102(0.0024-0.07305)$ & $0.00268(0.00046-0.02525)$ \\
\hline & SJR & & $1.06(0.55-6.14)$ & $0.93(0.24-1.68)$ \\
\hline \multirow[t]{2}{*}{ Publication fee } & Mandatory & & $5(31.3 \%)$ & $4(66.7 \%)$ \\
\hline & Optional & & $11(68.7 \%)$ & 2 Free $\mathrm{OA}^{* *}$ \\
\hline \multirow[t]{3}{*}{ Fee timing } & Before & & $0(0 \%)$ & $0(0 \%)$ \\
\hline & After & & $15(93.7 \%)$ & $4(66.7 \%)$ \\
\hline & Both & & $1(6.3 \%)$ & $0(0 \%)$ \\
\hline OA APC $(n=20)(U S D) * *$ & & & $3525(605-4700)$ & $2590(2300-2990)$ \\
\hline
\end{tabular}

OA: Open access, $\mathrm{C} / \mathrm{C}$ ratio: ratio of citations to $\mathrm{OA}$ articles to the ratio of citable OA articles in journals with hybrid OA publication policy. JIF: journal impact factor, CS: Citescore, HI: Hirsch index, SNIP: Source Normalized Impact per Paper, ES: Eigenfactor score, SJR: Scientific journal ranking. APC: Article Processing/Publishing Charge. USD: United States of America Dollars. $* p=0.03$, Mann-Whitney U test $* * 2$ journals are free of charge in full OA journals, 16 hybrid OA journals, and 4 journals in full OA have been compared. $p=0.007$, Mann-Whitney U test

Publisher's note Springer Nature remains neutral with regard to jurisdictional claims in published maps and institutional affiliations. 